\title{
Ingenol Mebutate vs. Daylight Photodynamic Therapy in a Kidney Transplant Recipient
}

\author{
Michael Mühlstädt \\ Division of Dermatology and Venerology, Geneva University Hospitals, Geneva, Switzerland
}

\section{Key Words \\ Ingenol mebutate · Photodynamic therapy - Daylight photodynamic therapy $\cdot$ Methyl aminolevulinate $\cdot$ Field cancerization $\cdot$ Actinic keratosis $\cdot$ Immunosuppression}

\begin{abstract}
We present the case of a 73-year-old male patient who had received a first renal transplant at 36 years and a second one at the age of 55 years. He is currently under immunosuppression with everolimus $2.5 \mathrm{mg} /$ day and prednisone $5 \mathrm{mg} /$ day. The patient presented with multiple actinic keratoses on both cheeks and the forehead and received treatment by ingenol mebutate $150 \mu \mathrm{g} / \mathrm{g}$ gel daily on 3 consecutive days on his right cheek and methyl aminolevulinate (MAL) photodynamic therapy activated by daylight (MAL-dPDT) on the forehead and the left cheek. MAL-dPDT treatment proved a feasible, repeatable, physician-directed method of treating field cancerization with limited morbidity for a period of 6 days. Treatment with ingenol mebutate gel was a feasible, possibly self-directed method of treating field cancerization with limited morbidity for 10 days in this immunosuppressed patient. Both treatments showed similar efficacy. At the time of treatment, the MAL daylight PDT ran at 3 times the cost of ingenol mebutate gel.

(c) 2016 S. Karger AG, Basel
\end{abstract}

This is the case of a 73-year-old male patient whom we follow on a regular basis in our outpatient clinic. At the age of 36 years, he received a first deceased-donor renal allograft for glomerular insufficiency of unknown origin and has been under continued immunosuppression ever since. Due to rejection of the first graft, he was transplanted a second time at the age of 55 years. Current immunosuppression employs everolimus $2.5 \mathrm{mg} /$ day and prednisone $5 \mathrm{mg} /$ day. The patient has a long-standing history of non-melanoma skin cancer including actinic keratoses, squamous cell carcinomas and basal cell carcinomas.

He has by now reached a state of pronounced field cancerization. Current guidelines prefer treatments that do not only remove a single lesion but will also address the already underlying cellular changes in its vicinity $[1,2]$. Results of any one of these therapies may have a high inter-patient variability. For this reason, we chose 2 fielddirected therapies - ingenol mebutate and photodynamic therapy (PDT) - in order to assess their individual efficacy in our patient.

We applied $150 \mu \mathrm{g} / \mathrm{g}$ ingenol mebutate gel once daily to the lesions on the right cheek on 3 consecutive days by respecting a maximum total surface area of $25 \mathrm{~cm}^{2}$ as described in the official protocol of the drug [3]. Figure 1 shows the untreated right cheek, figure 2 shows the cheek

\section{KARGER}

E-Mail karger@karger.com

www.karger.com/drm
(C) 2016 S. Karger AG, Basel

$1018-8665 / 16 / 2327-0020 \$ 39.50 / 0$
Michael Mühlstädt

Division of Dermatology and Venerology, Geneva University Hospitals Rue Gabrielle-Perret-Gentil 4

$\mathrm{CH}-1205$ Geneva (Switzerland)

E-Mail mmuh@hcuge.ch 


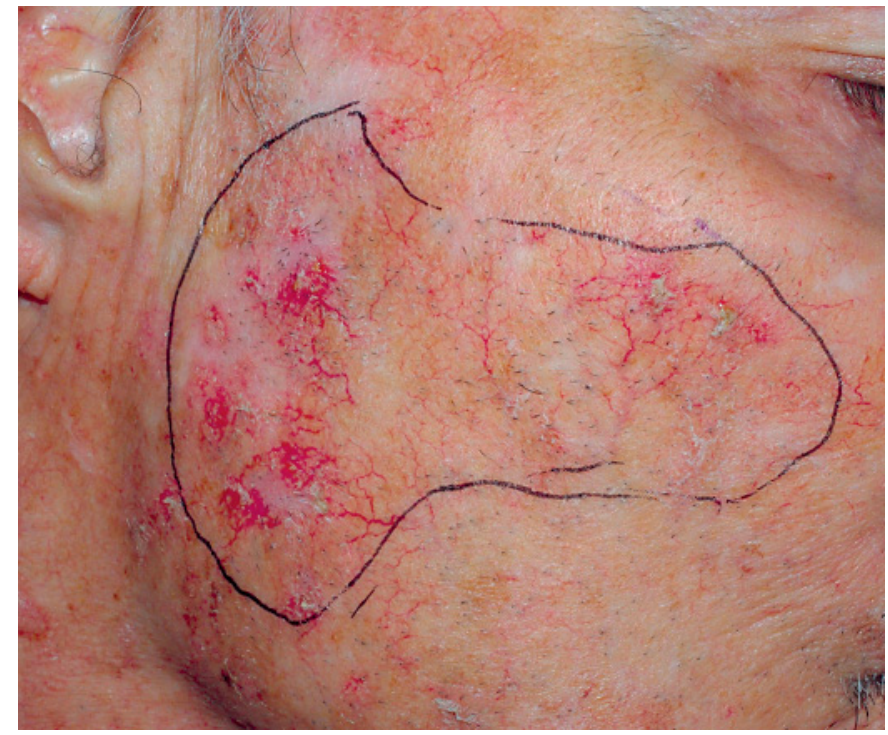

Fig. 1. Baseline of right cheek before treatment. The treatment area was marked with a pen. The patient left this labeling on for the 3 days of the treatment with ingenol mebutate.

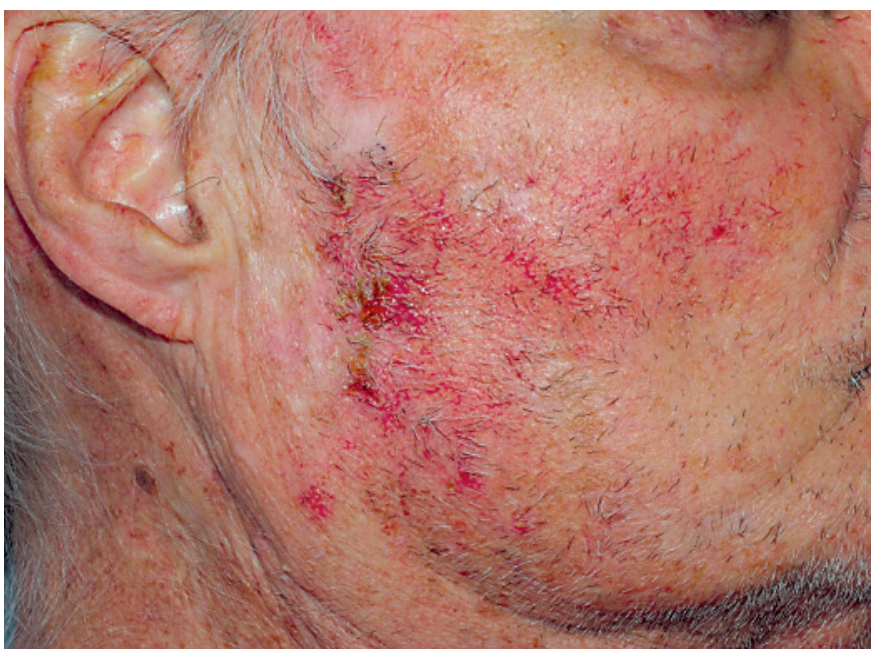

Fig. 3. Right cheek of the patient 10 days after the first application of ingenol mebutate $150 \mu \mathrm{g} / \mathrm{g}$ gel.

after 3 once daily applications of $150 \mu \mathrm{g} / \mathrm{g}$ ingenol mebutate gel as registered. A pustular reaction with crusts and erythema appeared in the gel treatment area. The patient reported only minor discomfort due to a mild burning sensation and had been informed about and consented orally to this not undesirable reaction beforehand. Ten days after the first application of ingenol mebutate

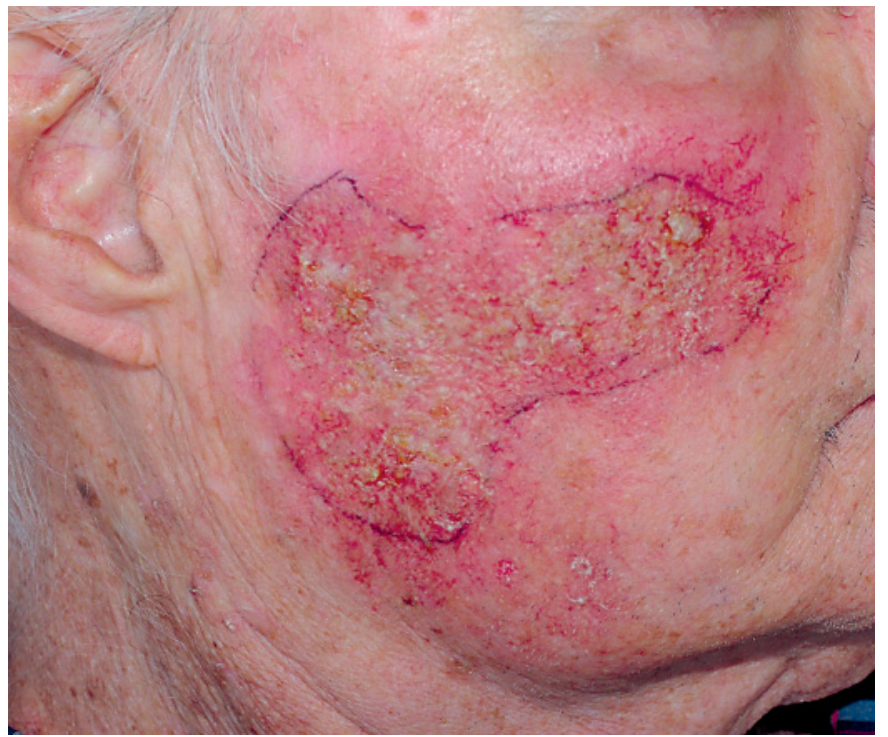

Fig. 2. Right cheek of the patient after 3 days of ingenol mebutate $150 \mu \mathrm{g} / \mathrm{g}$ once daily.

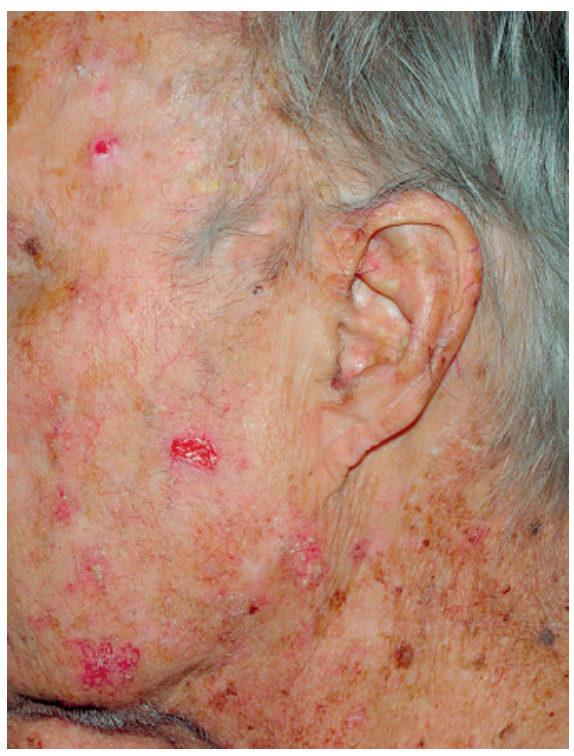

Fig. 4. Left cheek of the patient prior to MAL-dPDT treatment.

$150 \mu \mathrm{g} / \mathrm{g}$ gel to the lesions on the right cheek, we saw the patient again (fig. 3). Erythema had almost returned to baseline.

On the same day, we conducted a treatment of daylight activated PDT with methyl aminolevulinate (MALdPDT) on the left cheek (fig. 4: baseline of the left cheek before MAL-dPDT treatment) according to the following 


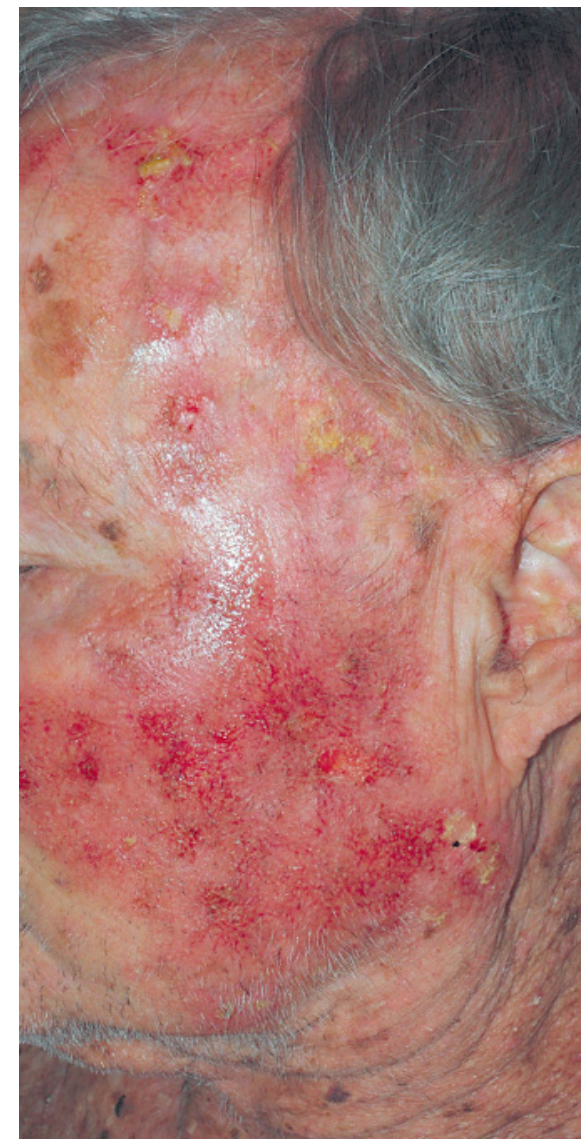

Fig.5. Left cheek of the patient immediately after $2 \mathrm{~h}$ of MAL-dPDT treatment.

protocol [4] - we applied chemical sunscreen to the face of the patient. After $30 \mathrm{~min}$, the hyperkeratotic lesions (actinic keratoses) on the left cheek and the forehead were prepared by light curettage. Then MAL 16\% was applied on the left cheek and the forehead. Directly afterwards, the patient spent $2 \mathrm{~h}$ in the hospital garden on a sunny day. After $2 \mathrm{~h}$, the remaining MAL was washed off and physical sunscreen was applied to all of the face (fig. 5: left cheek immediately after the MAL-dPDT treatment). The patient reported only minor discomfort during this treatment with a mild burning sensation. He then left our outpatient clinic and was instructed to go home immediately and spend the rest of the day inside in order to avoid further activation of protoporphyrin IX.

Sixteen days after the first ingenol mebutate treatment and hence 6 days after the MAL-dPDT treatment, we saw the patient again. Erythema had returned to normal levels on both sides. We observed comparable efficacy in the treatment of thin actinic keratoses (fig. 6: right cheek, 16 days after the beginning of a 3 day course of ingenol

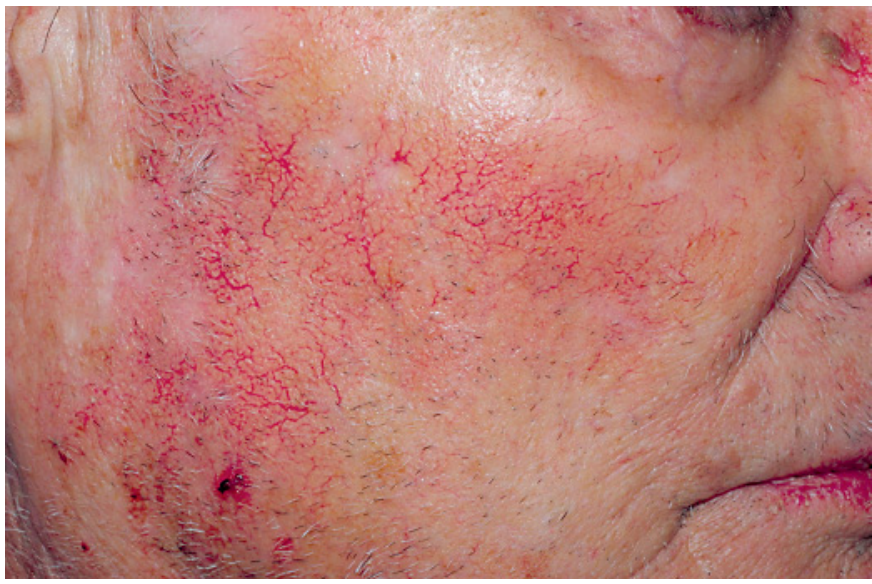

Fig. 6. Right cheek, 16 days after the beginning of a 3 days ingenol mebutate $150 \mu \mathrm{g} / \mathrm{g}$ gel treatment.

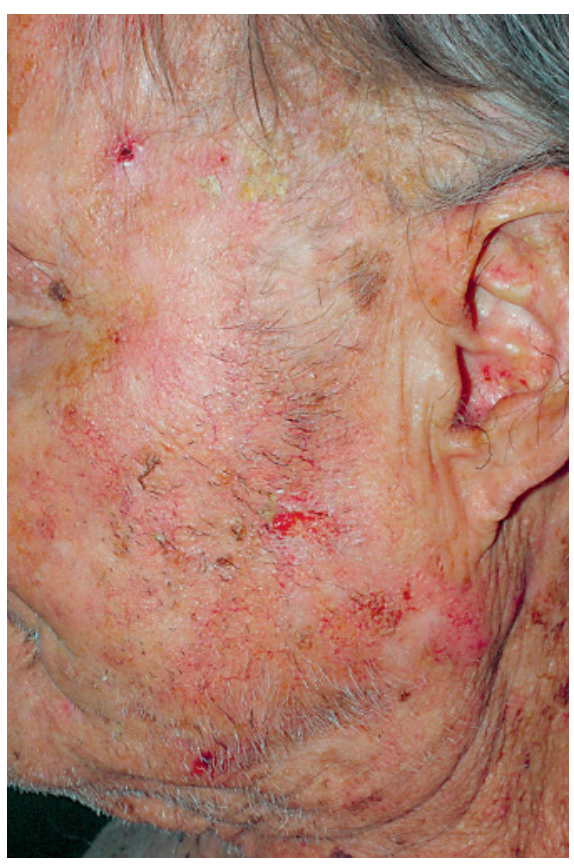

Fig. 7. Left cheek, 6 days after MAL-dPDT treatment.

mebutate $150 \mu \mathrm{g} / \mathrm{g}$ gel; fig. 7: left cheek, 6 days after MAL$\mathrm{dPDT}$ treatment). Please note that thicker plaques and ulcerations were unchanged by treatment. These are beyond the indications for either field treatment used and were thus excised afterward.

We present this case in order to demonstrate feasibility, document patient discomfort and clinical outcome of ingenol mebutate in comparison with MAL-dPDT. Treatment with ingenol mebutate does not necessitate any 
preparation before the application or any special care afterwards. The process of application is completed in about $30 \mathrm{~s}$ per day. If the patient is able to apply a cream to the site of the treatment, he/she will be able to perform the therapy by him/herself. If necessary, limits can be drawn with a pen in order to identify the area of treatment, as was the case in our patient. We recommend covering the zone of application by a simple dressing in order to reduce brushing against the zone and - in case of a facial lesion - to improve social appearance. It is imperative to explain the process of desired reactions including pustules and crusts, redness and oozing prior to the treatment. Colour tables provided by the manufacturer of the gel can be helpful. Our patient reported only minor discomfort after the treatment due to a mild burning sensation. Ten days after the first application, we saw only mild irritation left. We achieved complete clearance of actinic keratoses without discoloration of the skin 16 days after ingenol mebutate $150 \mu \mathrm{g} / \mathrm{g}$ gel therapy. This treatment is officially registered for a surface of up to $25 \mathrm{~cm}^{2}$ at a time [3].

For MAL-dPDT, preparation before the treatment (sunscreen, curettage) and special care after the treatment (washing the face, sunscreen, avoidance of further exposition to visible light) are necessary. This can, however, be done in one day. Post-treatment redness, crusting, pustules and oozing are similar to the ingenol mebutate treatment and must be addressed explicitly before the treatment. Our patient reported only mild discomfort due to a mild burning sensation. This is typical for the treatment and is generally well tolerated. We achieved complete clearance of thin actinic keratoses after 6 days, when irritation had returned to baseline.
MAL-dPDT treatment was a feasible, repeatable, physician-directed method of treating field cancerization with a limited down-time of around 6 days. Treatment with ingenol mebutate gel was a feasible, repeatable, physician-directed method of treating field cancerization with a down-time of around 10 days in this immunosuppressed patient. We expect this patient to undergo numerous treatments in the future as a consequence of his immunosuppression. He will be able to apply the ingenol mebutate treatment himself on the next occasion. At the time of treatment, the MAL-dPDT ran at 3 times the cost of ingenol mebutate gel.

\section{Acknowledgments}

The author would like to thank Dr. Evangelia Tzika (ingenol mebutate) and Dr. Donato Ferrara (MAL-dPDT) who were in charge of the treatments at the Division of Dermatology and Venereology, Geneva University Hospitals, Switzerland.

\section{Statement of Ethics}

The patient gave informed consent prior to the treatments.

\section{Disclosure Statement}

The author has previously received financial support for lectures and travel from LEO Pharma and Galderma Schweiz AG. Neither company was involved in the process of this article.

\section{References}

1 Werner RN, Stockfleth E, Connolly SM, Correia O, Erdmann R, Foley P, Gupta AK, Jacobs A, Kerl H, Lim HW, Martin G, Paquet M, Pariser DM, Rosumeck S, Röwert-Huber HJ, Sahota A, Sangueza OP, Shumack S, Sporbeck B, Swanson NA, Torezan L, Nast A: Evidence- and consensus-based (S3) guidelines for the treatment of actinic keratosis - international league of dermatological societies in cooperation with the European dermatology forum - short version. J Eur Acad Dermatol Venereol 2015;29: 2069-2079.
2 Hofbauer G, Anliker M, Boehncke WH, Brand C, Braun R, Gaide O, Hafner J, Hunger R, Itin P, Kaeuper G, Lautenschlager S, Mainetti C, Streit M: Swiss clinical practice guidelines on field cancerization of the skin. Swiss Med Wkly 2014;144:w14026.

3 Fachinformation: Picato ${ }^{\circledR} 150 \mathrm{mcg} / \mathrm{g}, \mathrm{Gel}$, Picato ${ }^{\circledR} 500 \mathrm{mcg} / \mathrm{g}$, Gel. http://www.swiss medicinfo.ch/ShowText.aspx? text Type= FI\&lang $=\mathrm{DE} \&$ authNr $=62763$

4 Gaide O, Hofbauer G, Hunger R, Mühlstädt M, Plaza T: Consensus on daylight photodynamic therapy (dl-PDT) in Switzerland. Derm Hel 2015;27:34-37. 